\title{
Community-based respiratory viral infections in HIV positive patients with lower respiratory tract disease: a prospective bronchoscopic study
}

\author{
R F Miller, C Loveday, J Holton, Y Sharvell, G Patel, N S Brink
}

Objectives: To evaluate the contribution of community-based respiratory virus infections to lower respiratory tract disease in HIV-1 infected individuals.

Design: Prospective clinical cohort study.

Setting: Specialist in-patient unit for HIV and AIDS, University College London Hospitals, London.

Subjects: 44 consecutive HIV-1 antibody positive patients who underwent 47 diagnostic bronchoscopies for evaluation of the symptoms and signs of lower respiratory tract disease.

Time: Winter months of 1994/95.

Main outcome measures: Detection, in bronchoscopic alveolar lavage fluid, of infection with influenza $A$ and $B$, respiratory syncytial virus (RSV), parainfluenza 1,2 and 3 (by immunofluorescence and cell culture) and adenovirus and enteroviruses (by cell culture).

Results: No evidence of influenza, RSV, parainfluenza, adenovirus, or enterovirus infection was detected.

Conclusions: Despite a marked increase in RSV and influenza B infection in the general population over the winter of 1994-95, respiratory virus infections were not detected in this cohort of HIV infected patients. As the organisms causing lower respiratory tract disease were related to immunosuppression, this study questions the value of routine identification of community-based respiratory viruses in this patient group.

(Genitourin Med 1996;72:9-11)

Keywords: HIV; respiratory infection; virus; bronchoscopy

Academic Department of Genitourinary

Medicine

R F Miller

Department of

Virology

C Loveday

Y Sharvell

G Patel

N S Brink

Department of

Microbiology

J Holton

Division of Pathology,

University College

London Medical

School, Camden \&

Islington Community

Health Services NHS

Trust and University

College London

Hospitals NHS Trust,

Middlesex Hospital

Site, London W1N 8AA

Correspondence to:

Dr R F Miller, First Floor

Crosspiece, Middlesex

Hospital, London

WIN 8AA, UK.

Accepted for publication

27 November 1995

\section{Introduction}

Up to $85 \%$ of symptomatic HIV infected individuals will have one or more episodes of lower respiratory tract disease, the vast majority of which are infectious in origin. ${ }^{1}$ Attention has been focused on the role of opportunistic pathogens, for example Pneumocystis carinii; however, the role of common respiratory pathogens such as influenza $A$ and $B$, respiratory syncytial virus, (RSV) and parainfluenza viruses clearly also need to be considered. The likelihood of respiratory complications following influenza infection is increased in patients with certain underlying conditions including cardiac and pulmonary disease and immunosuppression. ${ }^{2}$

In prospective cohort studies from the USA the influenza syndrome appears to occur with equal frequency in HIV-infected and uninfected individuals. ${ }^{3}$ HIV infected patients with influenza virus infection have a typical clinical presentation and rate of secondary complications, but may have more serious respiratory compromise and a prolonged duration of illness. ${ }^{4}$ However, it is not known how commonly influenza and other respiratory viruses contribute to lower respiratory tract disease in HIV infected patients in the UK. Currently the Department of Health in the United Kingdom $^{5}$ and the Immunisation Practices Advisory Committee of the Centres for Disease Control and Prevention (CDC) in the
USA $^{6}$ recommend yearly immunisation of HIV infected individuals against influenza. We have studied HIV-infected patients undergoing bronchoscopy for evaluation of lower respiratory tract disease during the winter months of 1994-95 in order to determine the frequency of infection with community-based respiratory viruses including influenza, RSV, parainfluenza, adenovirus and enterovirus. Our aim was to define the role of these agents in the pathogenesis of lower respiratory tract disease in a cohort of HIV-infected patients in London.

\section{Patients and methods}

We prospectively studied consecutive HIV-1 antibody positive patients presenting with symptoms, signs and/or chest radiographic abnormalities suggestive of lower respiratory tract disease who were admitted to the specialist in-patient unit for HIV/AIDS at University College London Hospitals, London. The study was conducted over a five month period of 1994-95 during the autumn and winter (01 1094 to 280295 ).

All patients underwent fibreoptic bronchoscopy and, following inspection of the tracheobronchial tree, alveolar lavage was carried out as previously described. ${ }^{7}$ All bronchoscopies were performed by one of us (RFM). Alveolar lavage fluid was analysed as follows: 
(1) Cytological examination for detection of Pneumocystis carinii

Lavage was smeared directly onto six slides and fixed immediately in $95 \%$ industrial methylated spirit (IMS), the remainder of the specimen was agitated for $10 \mathrm{~min}$ (or until liquefied) with an equal volume of $0.3 \%$ dithiothreitol in $0.02 \mathrm{M}$ EDTA at $\mathrm{pH} 7.0$ in a water bath at $37^{\circ} \mathrm{C}$ to achieve mucolysis. The specimen was then centrifuged for 5 minutes at $1500 \mathrm{rpm}$ to obtain a cell pellet which was smeared on six slides and fixed in IMS. Both preparations were stained by Grocott's methenamine silver and Papanicolou stains.

(2) Microbiological examination for detection of conventional bacterial pathogens and mycobacteria A sample of lavage was centrifuged for 10 minutes at $3000 \mathrm{rpm}$ and the spun deposit was used to prepare slides for Gram and Auramine staining. The remainder of the spun deposit was inoculated onto (a) chocolate agar and incubated in a $10 \%$ carbon dioxide atmosphere, (b) Cysteine Lactose Electrolyte Deficient medium (CLED, Oxoid CM3901), for 24 hours at $37^{\circ} \mathrm{C}$ (c) Sabouraud's medium and incubated in air for 14 days at $30^{\circ} \mathrm{C}$, and (d) $5 \%$ horse blood agar, with and without neomycin $(75 \mathrm{mcg} / \mathrm{ml})$, and incubated in anaerobic conditions. The second half of the specimen was decontaminated with $2 \%$ sodium hydroxide solution for 10 minutes, neutralised with phosphate buffer $\left(\mathrm{KH}_{2} \mathrm{PO}_{2}\right.$ $\left.36.0 \mathrm{~g} / 1 ; \mathrm{Na}_{2} \mathrm{HPO}_{4} 47 \mathrm{~g} / \mathrm{l}\right)$, centrifuged at $3000 \mathrm{rpm}$ for 10 minutes and inoculated onto Lowenstein Jensen medium. This was incubated for up to 12 weeks at $35^{\circ} \mathrm{C}$.

\section{(3) Virological examination}

The bronchoalveoloar lavage fluid was spun at $1000 \mathrm{rpm}$ and the cellular deposit spotted onto a slide (Multispot microscope slides, C A Hendley Ltd, Essex, England), dried and fixed in acetone. Direct immunofluorescence for influenza A and B, RSV and parainfluenza 1, 2 and 3 was then performed (Dako, Imagen ${ }^{\mathrm{TM}}$, Dako, Cambridgeshire, UK). A positive result

Diagnoses in 47 episodes of symptomatic lower respiratory tract disease in a total of 44 patients

\begin{tabular}{ll}
\hline Diagnosis & $n$ \\
\hline P carinii pneumonia & 26 \\
$\quad$ also had Streptococcus pneumoniae infection & \\
1 also had Staphylococcus aureus infection & \\
1 also had pulmonary Kaposi's sarcoma & \\
1 also had Cryptococtus neoformans & \\
Pulmonary Kaposi's sarcoma & 6 \\
$\quad$ also had C neoformans infection & \\
1 also had Aspergillus fumigatus infection & \\
Bacterial bronchitis & \\
$\quad$ also had Mycobacterium avium intracellulare infection & 8 \\
Bacterial pneumonia & \\
1 S aureus & 2 \\
Ps aeruginosa & \\
Lymphocytic pneumonitis & \\
Mycobacterium tuberculosis & 2 \\
Tracheitis & 1 \\
Self-limiting symptoms/negative investigations & 1 \\
Total & 1 \\
\hline
\end{tabular}

was indicated by identification of a typical pattern of immunofluorescence. In addition all samples were inoculated into culture tubes of primary Rhesus monkey kidney cells and human embryo lung fibroblasts. The tissue culture tubes were checked daily for the appearance of a viral cytopathic effect. Haemadsorption was performed on day 10 post inoculation using guinea pig red blood cells. The presence of suspected parainfluenza, RSV or influenza viruses was confirmed by immunofluorescence, and of adenovirus by electron microscopy. The presence of enteroviruses was confirmed by specific neutralisation in cell culture.

Bacterial pneumonia was diagnosed if the patient had fever and focal or diffuse radiographic abnormalities with or without purulent sputum and either positive culture of a bacterial pathogen from blood or alveolar lavage fluid (and negative results of silver staining) with response to specific antibiotics in conventional doses. ${ }^{8} \mathrm{~A}$ diagnosis of bronchitis was made if the patient had cough and dyspnoea and was expectorating purulent sputum with a normal chest radiograph, a negative silver stain of alveolar lavage fluid and response to antibiotics in conventional doses. ${ }^{8}$ Kaposi's sarcoma was diagnosed on the basis of visual identification at bronchoscopy of typical red or violaceous raised or plaque like lesions in the tracheobronchial tree. ${ }^{9}$

\section{Results}

There were 44 patients, including 41 men and three women. Thirty-nine of the 41 were homosexual, one was an injecting drug user and one was a homosexual and injecting drug user. The three women were heterosexual and of African origin. At the time of the study all were resident in North London. CD4 + lymphocyte counts ranged from 0 to 0.31 (median $=0.03) \times 10^{9} / 1$ (normal range $=0.35$ to 2.2 $\left.\times 10^{9} / 1\right)$. In all but three patients the CD4 + count was $<0.2 \times 10^{9} / 1$. One patient was admitted on three occasions and a second patient twice; thus in total there were 47 episodes investigated.

A specific diagnosis was made in 46 of the 47 episodes; 26 (55\%) of the patients had $P$ carinii pneumonia. One patient had self-limiting symptoms with a normal chest radiograph and no identifiable bacterial or viral pathogen. Results are shown in the table. No patient had evidence of infection with adenovirus, enterovirus, RSV, parainfluenza virus types 1 , 2 or 3 or influenza virus types A or B.

\section{Discussion}

In this prospective study our aim was to evaluate the contribution of respiratory virus infections to lower respiratory tract disease in a cohort of symptomatic HIV-1 infected individuals undergoing diagnostic bronchoscopy over the winter months of $1994 / 95$. Over the period of the study there was a marked increase in reports of RSV and influenza $B$ infection to the PHLS Communicable Disease 
Surveillance Centre compared with the equivalent months in the previous year. ${ }^{10}$ Despite these reports of infection in the general population we were unable to demonstrate evidence of infection with parainfluenza, adenovirus, enterovirus, RSV or influenza in our patients. In the majority of patients lower respiratory tract disease was a reflection of their immunosuppression and not of the seasonal variation in prevalence of respiratory pathogens.

Respiratory viruses, other than herpesviruses, have previously been found only rarely in HIV infected patients undergoing diagnostic bronchoscopy for evaluation of possible lower respiratory tract disease. In a retrospective study from the University of Cincinatti Medical Centre 1199 bronchoscopic alveolar lavage samples were obtained from 895 patients over a 6 year period. ${ }^{11}$ Ninety-five percent of patients were immunosuppressed, two thirds by HIV infection, the remainder by other causes including bone marrow transplantation, lymphoma, cancer and long-term steroid use. In $37(3.1 \%)$ of the lavage samples non-herpesviruses were identified including rhinovirus in 12, influenza in 11, parainfluenza in seven, adenovirus in five and RSV and coxsackie A9 in a single patient each. Re-analysis of the data from this study to include only those immunosuppressed patients with HIV infection, shows that of 751 lavage samples, adenovirus was identified in three (two also had $P$ carinii pneumonia and one had unspecified bacterial co-infection) parainfluenza was found in two (one also had $P$ carinii pneumonia and one had a bacterial infection), RSV in one (who also had Cryptococcus neoformans pneumonia) and coxsackie A9 in one. The overall infection rate was $0.9 \%$; neither rhinovirus nor influenza were identified in this sub-group. The absence of influenza in this HIV infected population, in contrast to those patients immunosuppressed by other causes, may have been due to the fact that the University of Cincinatti Medical Center had followed CDC recommendations ${ }^{6}$ and had an aggressive policy of immunising HIV positive patients against influenza.

The Department of Health guidelines state that influenza immunisation is "strongly recommended" for individuals with immunosuppression due to treatment or disease including HIV infection. ${ }^{5}$ Because of concerns about the efficacy of influenza vaccination in HIV infected individuals, one study showed no antibody response to influenza vaccine in patients with $\mathrm{CD} 4+$ lymphocyte counts $<0.10 \times 10^{9} / 1$ and a sub-optimal response in patients whose counts ranged from $\geqslant 0 \cdot 10-0.30 \times 10^{9} / 1,{ }^{12}$ our unit does not routinely offer immunisation against influenza to
HIV infected individuals, regardless of their CD4 + lymphocyte count. Thus immunisation is not the explanation for the absence of detectable influenza virus infection in our patients. Our study does, however, only evaluate the contribution of respiratory viral infections to severe lower respiratory tract disease requiring hospitalisation and diagnostic bronchoscopy and not those individuals presenting with milder upper and lower respiratory tract disease. We were particularly interested in this group of patients as the policy of influenza immunisation is specifically aimed at preventing complications associated with infection with the virus. This study will be extended over a further winter season in order to examine the influences of annual variations in climate conditions and circulating community-based respiratory viruses on the prevalence of lower respiratory tract disease in HIV infected patients in North London.

In conclusion, at a time when influenza B and RSV infections were prevalent in the general population, common respiratory viral pathogens were not identified in this small cohort of HIV infected individuals with lower respiratory tract disease. This questions the value of routine laboratory identification of community based respiratory viruses in this patient group.

We thank Jane Rutherford for typing the manuscript.

1 Murray JF, Felton CP, Garay SM. Pulmonary complications of the acquired immunodeficiency syndrome; report of a National Heart, Lung and Blood Institute workshop. N Engl F Med 1984;310:1682-8.

2 Glezen WP, Decker M, Perrotta DM. Survey of underlying conditions of persons hospitalised with acute respiratory disease during influenza epidemics in Houston 1978-81. Am Rev Respir Dis 1987;136:550-5.

3 Wallace JM, Rao AV, Glassroth J, et al. Respiratory illness in persons with human immunodeficiency virus infection. persons with human immunodeficie

4 Safrin S, Rush JD, Mills J. Influenza in patients with human immunodeficiency virus infection. Chest 1990;98 33-37.

5 HMSO, London. Immunisation against Infectious Diseases, 1992: Influenza pp. 95-99.

6 Prevention and control of Influenza: Part 1, Vaccines. Recommendations of the Advisory Committee of Immunisation Practices. Morbidity and Mortality Weekly Report, 14 May 1993 Vol 42. RR-6.

7 Miller RF, Kocjan G, Buckland J, et al. Sputum induction for the diagnosis of respiratory disease in HIV positive for the diagnosis of respiratory

8 Pitkin AD, Grant AD, Foley NM, Miller RF. Changing patterns of respiratory disease in HIV antibody positive patients in a referral centre in the UK between 1986-7 and 1990-1. Thorax 1993;48:201-4.

9 Hughes-Davies L, Kocjan G, Spittle MF, Miller RF. Occult alveolar haemorrhage in broncho-pulmonary Kaposi's sarcoma. $\mathcal{F}$ Clin Pathol 1992;45:536-7.

10 CDR Weekly Report 1995:5: No 1. Public Health Laboratory service, Communicable Disease Surveillance Centre, London.

11 Conolly MG, Baughman RP, Dohn MN et al. Recovery of viruses other than cytomegalovirus from bronchoalveolar

lavage fluid. Chest 1994;105:175-81.
12 Kroon FP, van Dissel JT, de Jong JC, van Furth $R$. Antibody response to influenza, tetanus and pneumococcal vaccines in HIV-seropositive individuals in relation to the number of CD4 lymphocytes. AIDS 1994;8:539-44. 